\title{
EDITORIAL
}

\section{ONE WORLD PROBLEM, ONE WORLD SOLUTION?}

\author{
Industry is becoming increasingly involved in partnerships with public organizations to discover \\ drugs for infectious diseases that primarily affect the developing world. But the experience \\ gained from such initiatives could also help tackle the pressing worldwide challenges for \\ antimicrobial drug discovery in general.
}

This year sees the thirtieth anniversary of the Special Programme for Research and Training in Tropical Diseases. Better known as the TDR, this programme was created to address the need for research into tropical diseases such as malaria and African trypanosomiasis, which represent a massive health problem in the developing world. Despite the size of the problem, the lack of potential financial return on investment in discovering new treatments for such diseases has contributed to the well-known ' $90 / 10$ split': $90 \%$ of the US $\$ 70$ billion spent worldwide each year on health-related $\mathrm{R} \& \mathrm{D}$ is reported to be directed at just $10 \%$ of the global disease burden.

The general withdrawal of industry from R\&D in tropical diseases in the 1970s clearly had a major impact; only 13 of the more than 1,300 new drugs introduced between 1975 and 1999 were for tropical diseases. Furthermore, research in tropical diseases consequently missed out on the technological advances, such as high-throughput screening, that were introduced in industry in the 1980s and 1990s. Perhaps reflecting this, a significant proportion of the success of the TDR during this period was the result of working in partnership with industry to repurpose drugs originally developed for other indications to treat tropical diseases. For example, eflornithine, which was developed as an anticancer drug, has been successfully introduced for treating African trypanosomiasis.

But the past 5 years or so have seen a considerable increase in interest in $\mathrm{R} \& \mathrm{D}$ for tropical diseases, fuelled by major investments from philanthropic organizations such as the Gates Foundation, coupled with growing reengagement of industry in the area. Building on the example set by the TDR, initiatives such as the Medicines for Malaria Venture are further establishing the 'virtual' public-private partnership (PPP) as a model for drug development in tropical diseases ${ }^{1}$. Integrating the expertise of industry in screening, chemistry and preclinical activities with the knowledge public organizations have about tropical diseases, the type of drugs required and how to conduct clinical studies in this field has already led to notable successes, some of which are discussed in the review on page 727 of this issue. Moreover, such initiatives could help apply recent technological advances in drug $R \& D$ to fully exploit the recent availability of the genome sequences of many of the most important pathogens.

Of course, the issue of insufficient return on R\&D investment to support market-driven drug discovery remains. And indeed, this is another area in which the experience of industry in managing the best 'portfolio' of projects from a large number of possibilities could help maximise the output from the funding that is available for PPPs. But perhaps just as important could be the experience gained in how to make PPP models work effectively.

As highlighted in the news story on page 704, big pharma has largely sidelined antibacterial research, leaving few potential drugs in the pipeline to combat the inevitable emergence of bacterial resistance to current agents. The urgency of the situation is reflected by the fact that three bills have been introduced in the US Congress this year already in an attempt to address the problem.

Even if all of these bills are passed, they will only be the first step in reversing the dearth of antibiotic R\&D. And although the genomes of major pathogenic bacteria are now available, it is quite possible that agents developed against the most promising novel targets might only have narrow-spectrum activity, limiting their potential market. The degree of market-driven incentive for new drug development targeting emerging pathogens such as SARS is also unclear. So, translating the experience gained in a similar situation with PPPs for tackling developing-world diseases to infectious diseases in general could be valuable in combating the global threat that these diseases pose.

1. Nwaka, S. \& Ridley, R. G. Virtual drug discovery and development for neglected diseases through public-private partnerships. Nature Rev. Drug Discov. 2, 919-928 (2003). 